\title{
Additional Local Therapy for Liver Metastases in Patients with Metastatic Castration-Resistant Prostate Cancer Receiving Systemic PSMA-Targeted Therapy
}

\author{
Robert Seifert ${ }^{1}$, Katharina Kessel ${ }^{1}$, Martin Boegemann ${ }^{2}$, Michael Köhler ${ }^{3}$, Wolfgang Roll ${ }^{1}$, Lars Stegger ${ }^{1}$, \\ Matthias Weckesser ${ }^{1}$, and Kambiz Rahbar ${ }^{1}$ \\ ${ }^{I}$ Department of Nuclear Medicine, University Hospital Münster, Münster, Germany; ${ }^{2}$ Department of Urology, University Hospital \\ Münster, Münster, Germany; and ${ }^{3}$ Department of Clinical Radiology, University Hospital Münster, Münster, Germany
}

\begin{abstract}
The aim of this study was to evaluate the efficacy of ${ }^{177}$ Lu-prostatespecific membrane antigen (PSMA)-617 ( ${ }^{177}$ Lu-PSMA) and selective internal radiation therapy (SIRT) for the treatment of liver metastases of castration-resistant prostate cancer. Methods: Safety and survival of patients with metastatic castration-resistant prostate cancer and liver metastases assigned to ${ }^{177}$ Lu-PSMA alone $(n=31)$ or in combination with $\operatorname{SIRT}(n=5)$ were retrospectively analyzed. Additionally, a subgroup $(n=10)$ was analyzed using morphologic and molecular response criteria. Results: Median estimated survival was 5.7 mo for ${ }^{177}$ Lu-PSMA alone and 8.4 mo for combined sequential ${ }^{177}$ Lu-PSMA and SIRT. ${ }^{177}$ Lu-PSMA achieved discordant therapy responses with both regressive and progressive liver metastases in the same patient (best vs. worst responding metastases per patient: $-35 \%$ vs. $+63 \%$ diameter change; $P<0.05$ ). SIRT was superior to ${ }^{177} \mathrm{Lu}-$ PSMA for the treatment of liver metastases (0\% vs. $56 \%$ progression). Conclusion: The combination of ${ }^{177}$ Lu-PSMA and SIRT is efficient and feasible for the treatment of advanced prostate cancer. ${ }^{177} \mathrm{Lu}-\mathrm{PSMA}$ alone seems to have limited response rates in the treatment of liver metastases.
\end{abstract}

Key Words: PSMA; prostate cancer; SIRT

J Nucl Med 2020; 61:723-728

DOI: 10.2967/jnumed.119.233429

\section{D} espite marked progress in recent years, the therapy of metastatic castration-resistant prostate cancer (mCRPC) remains a substantial clinical challenge, especially since liver metastases are still associated with poor overall survival $(1,2)$. This is partly caused by a dedifferentiation of the prostate cancer cells in liver metastases, a process that is called neuroendocrine transdifferentiation and is associated with poor survival $(3,4)$. Radioligand therapies targeting the prostate-specific membrane antigen (PSMA) have been applied to patients with advanced $\mathrm{mCRPC}$ in multiple studies (5-8). Among the variety of available radioligands, ${ }^{177} \mathrm{Lu}$-PSMA$617\left({ }^{177} \mathrm{Lu}-\mathrm{PSMA}\right)$ is most commonly used. Preliminary data suggest that ${ }^{177} \mathrm{Lu}-\mathrm{PSMA}$ extends progression-free and overall survival

Received Jul. 8, 2019; revision accepted Sep. 20, 2019.

For correspondence or reprints contact: Kambiz Rahbar, Department of Nuclear Medicine, University Hospital Muenster, Albert Schweitzer Campus

1, Gebäude A1, 48149 Muenster, Germany.

E-mail: rahbar@uni-muenster.de

Published online Oct. 10, 2019.

COPYRIGHT (C 2020 by the Society of Nuclear Medicine and Molecular Imaging. while exhibiting a favorable toxicity profile $(5,9,10)$. However, ${ }^{177} \mathrm{Lu}-\mathrm{PSMA}$ seems to be less efficacious for the treatment of liver and other visceral metastases (5). In contrast, selective internal radiation therapy (SIRT) specifically targets liver metastases with great efficacy and therefore represents a treatment option for unresectable primary liver cancer or metastases in the liver (11). To date, however, there is no systematic evaluation of ${ }^{177} \mathrm{Lu}$-PSMA therapy or SIRT as a local liver therapy for liver metastases in advanced prostate cancer. Therefore, the aim of the present retrospective single-center study was to evaluate the efficacy of ${ }^{177} \mathrm{Lu}$-PSMA and SIRT in mCRPC patients with liver metastases and elucidate the implications for overall survival.

\section{MATERIALS AND METHODS}

\section{Patients}

Figure 1 presents the flowchart for patient selection. All patients with $\mathrm{mCRPC}$ and liver metastases referred to the Department of Nuclear Medicine for therapy were considered for this case series $(n=36)$. Patients with metastases were treated with PSMA-targeted therapy alone $(n=31)$ or in combination with SIRT $(n=5)$ based on individual tumor board decisions and with informed consent (Supplemental Table 1; supplemental materials are available at http://jnm.snmjournals.org). SIRT was pursued if the extrahepatic tumor burden was controlled but the hepatic metastases were progressive (no overall therapy failure). Under this criterion, only 5 patients were eligible to be treated with SIRT. Toxicity was evaluated according to the Common Toxicity Criteria for Adverse Events (CTCAE, version 5.0).

The following 2 inclusion criteria were applied for the subgroup analysis: the presence of liver metastases and the availability of PET imaging before and after radionuclide therapy. All 10 patients analyzed in detail received ${ }^{177} \mathrm{Lu}$-PSMA therapy; 4 patients were additionally treated with SIRT because of hepatic progress despite controlled extrahepatic tumor burden (Supplemental Table 2). The retrospective analysis of patients treated with ${ }^{177} \mathrm{Lu}$-PSMA was approved by the local ethics committee (approval 2016-585-f-S, Ethikkommission der Ärztekammer Westfalen-Lippe und der Westfälischen WilhelmsUniversität Münster).

\section{PSMA-Targeted Radionuclide Therapy}

Conjugation of PSMA-617 with ${ }^{177} \mathrm{Lu}$ has been described previously (12). The $\beta$-radiation of ${ }^{177} \mathrm{Lu}$ (maximum energy, $0.497 \mathrm{MeV}$ ) has a maximum tissue range of $2 \mathrm{~mm}$ (13). PSMA-617 was manufactured by ABX GmbH. Whole-body scintigraphy was performed after $48 \mathrm{~h}$ to monitor the retention of ${ }^{177} \mathrm{Lu}$-PSMA in prostate cancer metastases. An administered target activity of 6-7.5 MBq was used per cycle, and 


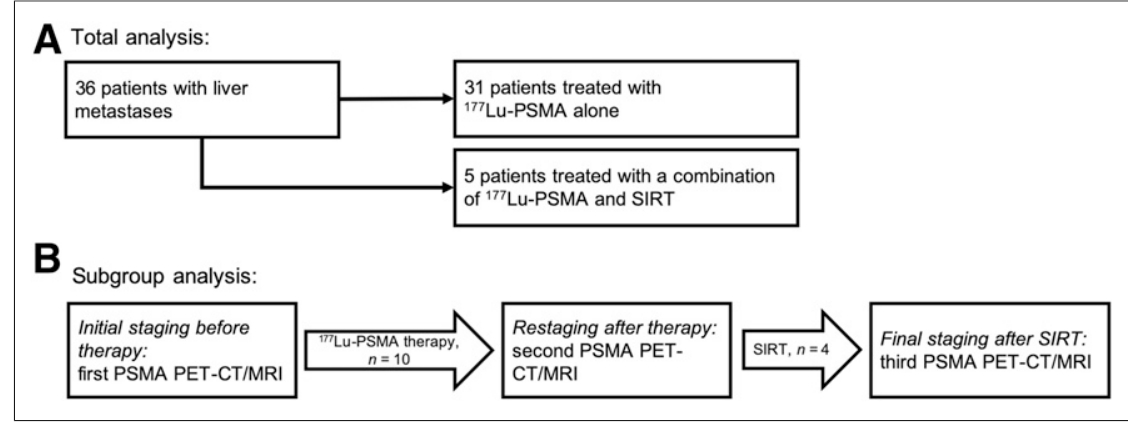

FIGURE 1. (A) Flowchart of patient selection for overall survival analysis. (B) Examination sequence for subgroup analysis (PSMA PET/CT or MRI before radionuclide treatment $=$ baseline, after ${ }^{177} \mathrm{Lu}-\mathrm{PSMA}=$ restaging, and after SIRT $=$ final staging).

the cycles were repeated after 7-8 wk. Exclusion criteria for ${ }^{177} \mathrm{Lu}-$ PSMA were a leukocyte level of less than $2,000 / \mu \mathrm{L}$, a platelet level of less than $75,000 / \mu \mathrm{L}$, a hemoglobin level of less than $8 \mathrm{~g} / \mathrm{dL}$, a creatinine level of more than $2 \mathrm{mg} / \mathrm{dL}$, and aspartate and alanine transaminase levels greater than 5 times the upper limit of normal (14).

\section{SIRT}

After selective catheterization, ${ }^{90} \mathrm{Y}$ microspheres were directly injected into the hepatic artery or its branches to cause radioembolization of metastases. Microspheres were manufactured by SIRTEX Medical. The $\beta$-particles of ${ }^{90} \mathrm{Y}$ (maximum energy, $2.27 \mathrm{MeV}$ ) have a maximum tissue range of $11 \mathrm{~mm}(13)$. The administered SIRT activity was calculated according to European Association of Nuclear Medicine guidelines (dependent on body surface area and relative tumor volume) (15). Exclusion criteria for radioembolization were the presence of ascites, elevated levels of bilirubin (cutoff, $2.0 \mathrm{mg} / \mathrm{dL}$ ), a hepatopulmonary shunt of more than $20 \%$

TABLE 1

Patient Characteristics

\begin{tabular}{|c|c|c|c|}
\hline Parameters & LM PSMA alone & LMPSMA+SIRT & All patients \\
\hline Patient count $(n)$ & 31 & 5 & 36 \\
\hline Age $(y)$ & $71.9(66.9-73.7)$ & $73.8(64.8-78.7)$ & $72.4(67.6-73.5)$ \\
\hline Gleason score & 8 & 8 & 8 \\
\hline Median PSA baseline (ng/mL) & $363.0[4.9-6,970]$ & $49.5[5.9-1,199]$ & $355.5[4.9-6,970]$ \\
\hline Median alkaline phosphatase (U/L) & $229.0[47-1,028]$ & $81.0[70-355]$ & $223.5[47-1,028]$ \\
\hline Median lactate dehydrogenase (U/L) & $448[160-7,802]$ & 389 [192-442] & $435[160-7,802]$ \\
\hline \multicolumn{4}{|l|}{ PSMA radioligand therapy } \\
\hline Average number of cycles & $2(2.0-3.6)$ & $3(2.7-4.0)$ & $2.5(2.2-3.6)$ \\
\hline Cycles total & 89 & 17 & 106 \\
\hline Average duration interval & $7.7(6.5-8.5)$ & $7.5(2.9-16.9)$ & $7.5(6.7-9.3)$ \\
\hline Average activity (GBq) & $6.2(6.1-6.4)$ & $6.4(5.7-7.5)$ & $6.2(6.2-6.5)$ \\
\hline \multicolumn{4}{|l|}{ ECOG PS $(n)$} \\
\hline $0-1$ & $21(67.8 \%)$ & $4(80 \%)$ & $25(69.4 \%)$ \\
\hline 2 & $8(25.8 \%)$ & $1(20 \%)$ & $9(25.0 \%)$ \\
\hline 3 & $2(6.4 \%)$ & $0(0 \%)$ & $2(5.0 \%)$ \\
\hline \multicolumn{4}{|l|}{ Site of metastases $(n)$} \\
\hline Bone & $31(100 \%)$ & $3(60 \%)$ & 34 (94.4\%) \\
\hline Lymph node & $24(77.4 \%)$ & $4(80 \%)$ & 28 (77.7\%) \\
\hline Lung & $8(25.8 \%)$ & $0(0 \%)$ & $8(22.2 \%)$ \\
\hline Other & $1(3.2 \%)$ & $0(0 \%)$ & $1(2.7 \%)$ \\
\hline \multicolumn{4}{|l|}{ Previous therapy for mCRPC $(n)$} \\
\hline Docetaxel & 29 (93.5\%) & $3(60 \%)$ & $32(88.8 \%)$ \\
\hline Cabazitaxel & $14(45.2 \%)$ & $2(40 \%)$ & $16(44.4 \%)$ \\
\hline Abiraterone & $26(83.8 \%)$ & $4(80 \%)$ & $30(83.3 \%)$ \\
\hline Enzalutamide & $25(80.6 \%)$ & $4(80 \%)$ & $29(80.5 \%)$ \\
\hline Abiraterone and enzalutamide & $22(70.1 \%)$ & $3(60 \%)$ & $25(69.4 \%)$ \\
\hline${ }^{223} \mathrm{Ra}$ & $6(19.3 \%)$ & $1(20 \%)$ & 7 (19.4\%) \\
\hline EBRT, bone & 17 (54.8\%) & $2(40 \%)$ & $19(52.8 \%)$ \\
\hline
\end{tabular}

$\mathrm{LM}_{\mathrm{PSMA} \text { alone }}=$ liver metastasis-only PSMA therapy; $\mathrm{LM}_{\mathrm{PSMA}+\mathrm{SIRT}}=$ liver metastasis PSMA therapy and SIRT; PSA = prostate-specific antigen; ECOG PS = Eastern Cooperative Oncology Group performance status; EBRT = external-beam radiation therapy.

Data in parentheses are $95 \% \mathrm{Cls}$ or percentages; data in square brackets are ranges. 
(assessed by ${ }^{99 m} \mathrm{Tc}$-macroaggregated albumin [ $\left.{ }^{99 \mathrm{~m}} \mathrm{Tc}-\mathrm{MAA}\right]$ scintigraphy), or persisting blood flow from the hepatic arteries to the gastrointestinal tract (evaluated by ${ }^{99 m}$ Tc-MAA scintigraphy and angiography) $(16,17)$. Patients were evaluated by angiography and injection of ${ }^{99 \mathrm{~m}} \mathrm{Tc}-\mathrm{MAA}$ into the hepatic arteries $2 \mathrm{wk}$ before the SIR treatment. SPECT and ${ }^{99 \mathrm{~m}} \mathrm{Tc}-$ MAA scintigraphy were used to estimate the relative hepatic tumor volume and to determine whether the metastases were hypervascularized. All lesions showed an accumulation of ${ }^{99 \mathrm{~m}} \mathrm{Tc}-\mathrm{MAA}$ at least at the margin and also within the target lesions. An example is given in Supplemental Figure 1. Toxicity after SIRT and ${ }^{177}$ Lu-PSMA therapy was evaluated using Common Terminology Criteria for Adverse Events (version 5).

\section{Imaging}

Whole-body staging (vertex to proximal tibia) was done using PSMA-based PET imaging before the start of ${ }^{177}$ Lu-PSMA therapy (initial staging), after the last cycle of ${ }^{177}$ Lu-PSMA (restaging), and after SIRT, so that the therapy effect could be clearly attributed to the precedent therapy. Contrast-enhanced MRI or CT was performed.

The Department of Nuclear Medicine switched from ${ }^{68} \mathrm{Ga}-\mathrm{PSMA}-$ 11 to ${ }^{18}$ F-PSMA-1007 for logistic reasons during the interval of this study. Therefore, whole-body staging was done using either tracer if no external acquisition was present. For ${ }^{68} \mathrm{Ga}$-PSMA-11-PET, $2 \mathrm{MBq}$ per kilogram of body weight were injected, and the PET acquisition took place $60 \mathrm{~min}$ after tracer administration (the ${ }^{68} \mathrm{G}$ was generated using a GalliaPharm [Eckert and Ziegler]; the PSMA-11 precursor was manufactured by $\mathrm{ABX} \mathrm{GmbH}$ ). For ${ }^{18}$ F-PSMA-1007, 4 MBq per kilogram of body weight were injected, and the scans were acquired $120 \mathrm{~min}$ after tracer administration (tracer synthesis was performed using a TracerLab MX synthesizer [GE Healthcare]; PSMA-1007 precursor was provided by $\mathrm{ABX} \mathrm{GmbH}]$ ).

A Biograph mMR PET/MRI system or a Biograph mCT PET/CT system was used to sequentially acquire PET/MRI and PET/CT images (Siemens Healthcare). Whole-body MRI comprised an axial T2-weighted HASTE (half-Fourier acquisition single-shot turbo spin-echo), an axial and coronal T1-weighted VIBE (volumetric interpolated breath-hold examination) with fat suppression (after injection of a gadoliniumcontaining contrast agent), and an axial T2-weighted turbo spin-echo sequence of the liver. Contrast-enhanced abdominal CT or MRI was performed using standard parameters.

\section{Response Evaluation}

Estimated mean overall survival was used as the primary endpoint to compare PSMA-targeted therapy alone $(n=31)$ or in combination with SIRT ( $n=5$ ). Additionally, the response to SIRT or ${ }^{177} \mathrm{Lu}$-PSMA was assessed using morphologic and molecular features in the subgroup analysis $(n=10)$. Morphologic response was assessed in analogy to RECIST, version 1.1, and was reported only for liver metastases: up to 4 of the largest hypervascularized hepatic metastases were defined as target lesions, and the sum of their long-axis diameters (LADs) was measured (progressive disease $=20 \%$ increase of LADs or newly formed metastases, partial response $=30 \%$ decrease of LADs, stable disease $=$ neither progressive disease nor partial response, and complete response $=$ no lesions definable) (18). $\mathrm{SUV}_{\max }$ was measured at baseline in all liver metastases to determine whether ${ }^{177} \mathrm{Lu}$-PSMA therapy was feasible. Additionally, the change in PSMA expression caused by SIRT was analyzed as a molecular response.

\section{Statistical Analysis}

SPSS statistics, version 25 (IBM), was used for testing and descriptive statistics. Unpaired (Mann-Whitney U) and paired (Wilcoxon) nonparametric statistical tests were performed. Values are presented as median; the range or confidence intervals (CIs) are additionally specified. A statistically significant difference was assumed if the $P$ value was less than 0.05 ; otherwise, nonsignificance is reported. The Kaplan-Meier method was used to estimate the median survival, and the log-rank method was used to test for survival differences.

\section{RESULTS}

\section{Patient Characteristics and Therapies}

All patients were treated with androgen deprivation therapy (Table 1). One additional patient was evaluated for SIRT but could not receive treatment because, in comparisons with previous imaging, the disease was found to be rapidly progressing. The average interval between ${ }^{177} \mathrm{Lu}-\mathrm{PSMA}$ administrations was $7.5 \mathrm{wk}$ (95\% CI, 6.7-9.3 wk); a median of 3 therapy cycles was administered, and the average dose was $6.2 \mathrm{GBq}$ (95\% CI, 6.2-6.5 GBq). A mean dose of $2.2 \mathrm{GBq}$ of ${ }^{90} \mathrm{Y}$ was used (range, $\left.1.3-2.5 \mathrm{GBq}\right)(n=5)$.

\section{Survival}

The estimated median overall survival $(n=31)$ was 5.7 mo (95\% CI, 2.9-8.5 mo) for patients receiving only ${ }^{177} \mathrm{Lu}-\mathrm{PSMA}$. For patients receiving ${ }^{177} \mathrm{Lu}-\mathrm{PSMA}$ and SIRT $(n=5)$, the estimated median overall survival was 8.4 mo (95\% CI, 4.9-11.9 mo). The estimated overall survival for ${ }^{177} \mathrm{Lu}$-PSMA and SIRT was not significantly longer than that of ${ }^{177} \mathrm{Lu}-\mathrm{PSMA}$ alone (log rank $P=0.227$ ).

\section{Subgroup Analysis of Efficacy of PSMA-Targeted Therapy}

Treated by ${ }^{177}$ Lu-PSMA therapy, liver metastases showed progressive disease compared with the baseline examination in $56 \%$ of patients; however, $44 \%$ displayed stable disease (9 patients; patient 3 was not considered here because initial SIRT was followed by ${ }^{177} \mathrm{Lu}$-PSMA therapy). Liver metastases had a discordant response: the best- and worst-responding lesions were significantly different if multiple liver metastases were present (only patients with multiple liver

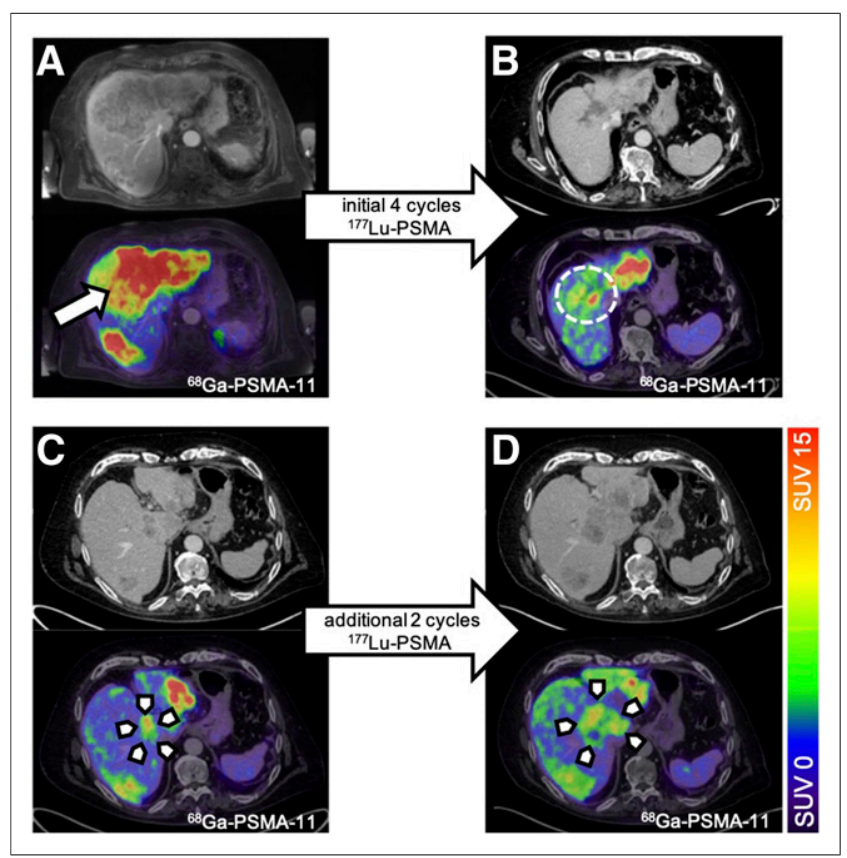

FIGURE 2. Discordant response of liver metastases to ${ }^{177} \mathrm{Lu}$-PSMA in patient 6 , who was treated with 6 cycles of ${ }^{177}$ Lu-PSMA. Shown are CT or MRI (top) and PET/CT or MRI (bottom) images. (A) Before start of ${ }^{177} \mathrm{Lu}-\mathrm{PSMA}$ therapy, major liver metastases were seen in both lobes (arrow). (B) After treatment with 4 cycles of ${ }^{177}$ Lu-PSMA, metastases were decreasing (dashed circle). (C) Small liver metastases were also detected in segment V. (D) However, after 2 additional cycles, metastases were progressive and showed only modest PSMA uptake caused by dedifferentiation (arrowheads). 


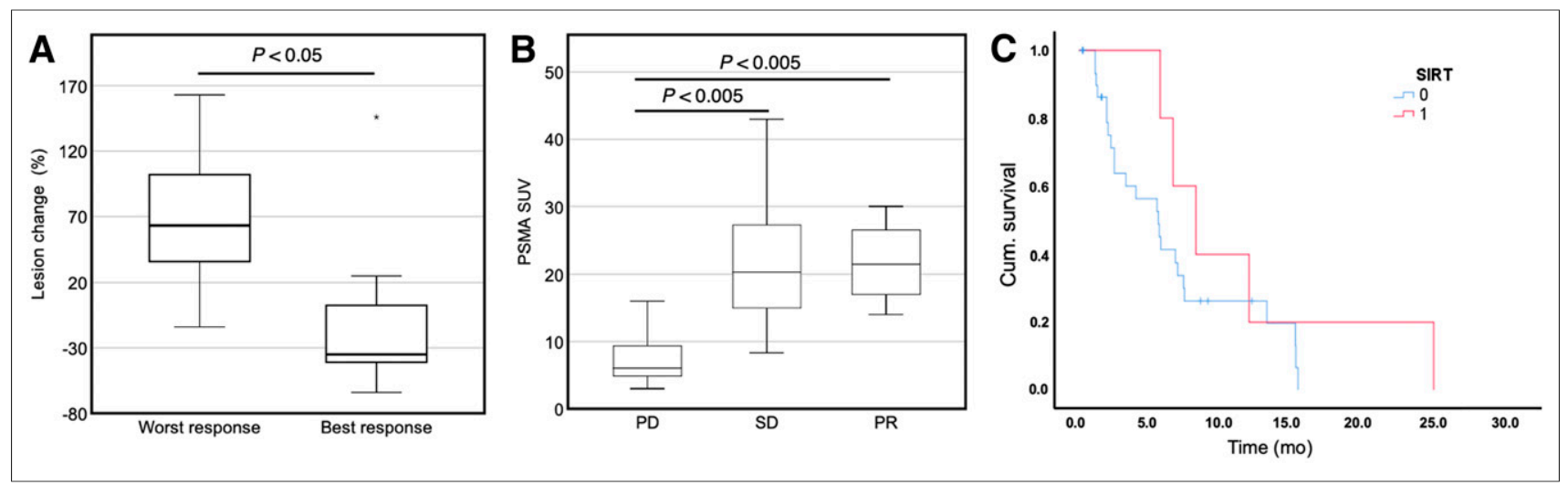

FIGURE 3. Evaluation of ${ }^{177}$ Lu-PSMA and SIRT efficacy. (A) ${ }^{177}$ Lu-PSMA achieved only discordant responses by liver metastases; therefore, changes in size for best- and worst-responding lesions were compared (only patients with multiple liver metastases at baseline were included [ $n=7]$ ). (B) Overall, liver metastases responded significantly differently to ${ }^{177}$ Lu-PSMA therapy. Treatment with ${ }^{177}$ Lu-PSMA therapy was efficient only in patients

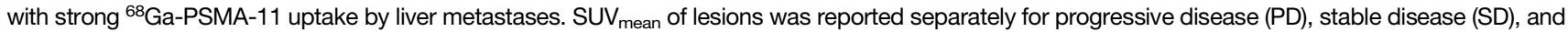
partially responding (PR) liver metastases (only patients with ${ }^{68} \mathrm{Ga}$-PSMA-11 radionuclide were included $[n=8]$ ). (C) Overall survival of patients treated with ${ }^{177}$ LuPSMA alone (median, $5.7 \mathrm{mo}$; blue) did not significantly differ from that of patients treated with combination of ${ }^{177}$ Lu-PSMA and SIRT (median, 8.4 mo; red).

metastases at baseline were included $[n=7] ;+63 \%$ vs. $-35 \%$ LAD change $[P<0.05]$; Figs. 2 and 3).

When the liver metastases of all patients were rated individually, morphologically progressive lesions showed significantly less
${ }^{68} \mathrm{Ga}$-PSMA-11 uptake at baseline than did stable and regressive ones (only patients with ${ }^{68} \mathrm{Ga}$-PSMA-11 radionuclide were included $\left[n=8\right.$ ]; $\mathrm{SUV}_{\max }$ of 6.1 for progressive disease, 20.3 for stable disease, and 21.5 for partial response [ $P<0.005$ ]; Fig. 3).
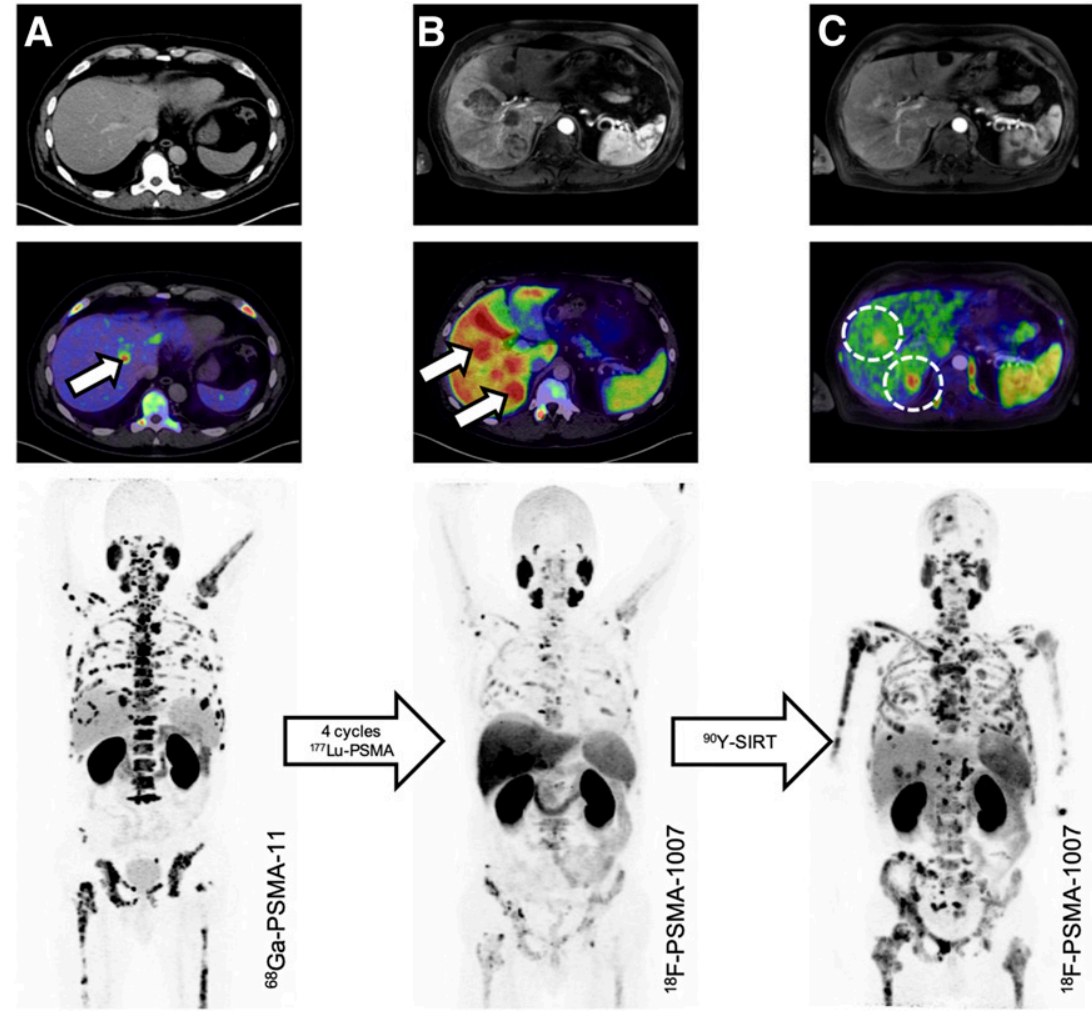

FIGURE 4. Response of liver metastases to ${ }^{177}$ Lu-PSMA and SIRT in patient 1, who was treated with 4 cycles of ${ }^{177}$ Lu-PSMA initially, followed by SIRT. Shown are CT (top), PET/CT (middle), and whole-body maximum-intensity projection (bottom) images before therapy (A), after 4 cycles of ${ }^{177}$ Lu-PSMA (B), and after ${ }^{90}$ Y-SIRT (C). Compared with pretherapy scan, liver metastases were progressive after ${ }^{177}$ Lu-PSMA and extrahepatic metastases were regressive (arrows). After SIRT, liver metastases had responded and had no relevant PSMA expression (dashed cycles). However, extrahepatic metastases were severely progressive, as can be seen on the maximum-intensity projection.

\section{Subgroup Analysis of Efficacy of SIRT}

The mean follow-up time point was 9 wk after SIRT (range, 3-13 wk; Fig. 4). When morphologic criteria were applied, $75 \%$ showed a partial response ( 3 patients) and $25 \%$ had stable disease ( 1 patient). The best- and worst-responding lesions did not significantly differ ( $n=4 ;-19 \%$ vs. $-44 \%$ LAD change).

The molecular response evaluation revealed that PSMA uptake by liver metastases was reduced by a median of $39 \%$ after SIRT (range, $+4 \%$ to $-83 \%$ ).

\section{Toxicity}

The hepatic toxicity of ${ }^{177} \mathrm{Lu}$-PSMA therapy alone versus ${ }^{177}$ Lu-PSMA therapy in combination with SIRT is presented in Supplemental Tables 3 and 4 according to the Common Terminology Criteria for Adverse Events (version 5). Briefly, there were significant differences in alanine aminotransferase ( $+160.0 \%$ vs. $+14.6 \% ; P=0.006)$, aspartate aminotransferase $(+130.3 \%$ vs. $+25.42 \%$; $P=0.007)$, and $\gamma$-glutamyltransferase $(+652.1 \%$ vs. $+2.6 \% ; P=0.0003)$ between ${ }^{177} \mathrm{Lu}-\mathrm{PSMA}$ and SIRT versus ${ }^{177} \mathrm{Lu}$-PSMA alone, but there was no significant difference in bilirubin.

\section{DISCUSSION}

The aim of this study was to evaluate the efficacy and safety of ${ }^{177} \mathrm{Lu}-\mathrm{PSMA}$ and SIRT for the treatment of advanced MCRPC with liver metastases, compared with ${ }^{177} \mathrm{Lu}$-PSMA alone. Patients treated with ${ }^{177}$ Lu-PSMA 
showed a discordant response to therapy, with both regressing and progressing metastases at the same time. The reason for this discordance currently remains unclear. Even initial responses to PSMA therapy may be misleading for a long-term response prediction. Initial responses to ${ }^{177} \mathrm{Lu}-\mathrm{PSMA}$ may not be associated with long-term remission, as newly occurring metastases might not respond to additional ${ }^{177} \mathrm{Lu}-\mathrm{PSMA}$ cycles. This behavior might partly be explained by the neuroendocrine transdifferentiation of prostate cancer cells, which is associated with poor overall survival $(3,19)$. In the transdifferentiation process, neuroendocrine cell markers (such as the neuron-specific enolase) are expressed, whereas adenocarcinoma markers (such as the prostate-specific antigen) are lost (19). The neuroendocrine differentiation is frequently present in liver metastases, which might partially explain the occurrence and progression of PSMA-negative metastases under ${ }^{177}$ Lu-PSMA therapy that was shown in the present study (3). However, the fact that patients treated with SIRT did not show a discordant hepatic response indicates its superiority for the treatment of liver metastases. This superiority might be due to the independence of SIRT from target molecules; therefore, neuroendocrine differentiation should not decrease its efficacy. Moreover, initial treatment of liver metastases with SIRT might prevent the spread of neuroendocrine differentiated tumor cells to other organs.

SIRT obtained higher response rates and a longer mean overall survival for the treatment of liver metastases than ${ }^{177} \mathrm{Lu}$-PSMA. The mean SIRT-delivered tumor dose for colorectal metastases is 55 Gy (20). ${ }^{177}$ Lu-PSMA therapy delivers mean tumor dosages of $32 \mathrm{~Gy}$ (for $6 \mathrm{GBq}$ of ${ }^{177} \mathrm{Lu}$ ) (21). For colorectal metastases, a deposition of at least $60 \mathrm{~Gy}$ is favorable for prolonged overall survival (20). Despite the fundamental difference in tumor biology between prostate cancer and colorectal cancer, the distinct efficacy of ${ }^{177} \mathrm{Lu}$-PSMA and SIRT might be partially explained by the delivered doses.

The increase in aspartate aminotransferase, alanine aminotransferase, and $\gamma$-glutamyltransferase levels was significantly higher in the SIRT group than in patients treated with ${ }^{177} \mathrm{Lu}$-PSMA alone. However, baseline and posttherapeutic liver enzymes were compared in order to evaluate the overall toxicity. Therefore, the increase in liver enzymes is at least partly attributable to the progression of hepatic metastases rather than to hepatotoxicity. Moreover, the increase in bilirubin levels was not significantly different.

The limitations of the present study comprise the retrospective data analysis, small patient cohort, and potential selection bias. However, liver metastases are an end-stage phenomenon of prostate cancer and thus generally rare. Therefore, the presented initial results are valuable and have direct implications for the treatment of prostate cancer and liver metastases, especially in cases of castration resistance and ${ }^{177} \mathrm{Lu}$-PSMA evaluation. In the present article, SIRT was the only evaluated local treatment for the liver metastases. Therefore, future studies should investigate whether other local therapies of liver metastases, such as transcatheter arterial chemoembolization or extracranial stereotactic radiotherapy, are efficient as well. An additional limitation is the efficacy assessment of ${ }^{177} \mathrm{Lu}$-PSMA and SIRT therapy, which was based on morphologic features. Future studies should consider additional imaging modalities such as ${ }^{18}$ F-FDG PET or diffusion-weighted MRI for the evaluation of treatment response, especially in the context of neuroendocrine transdifferentiation (22).

\section{CONCLUSION}

The combination of ${ }^{177}$ Lu-PSMA and SIRT is efficacious and feasible for the treatment of liver metastases in advanced mCRPC. ${ }^{177} \mathrm{Lu}$-PSMA alone seems inferior to SIRT for the treatment of liver metastases.

\section{DISCLOSURE}

Kambiz Rahbar reports being a compensated consultant for $\mathrm{ABX}$ and has received consultant fees from Bayer and lectureship fees from Janssen Cielag, Amgen, AAA, and SIRTEX. The University of Münster received consulting fees from $\mathrm{ABX} \mathrm{GmbH}$, Radeberg, Germany, for Kambiz Rahbar and Martin Boegemann. No other potential conflict of interest relevant to this article was reported.

KEY POINTS

QUESTION: Is the combination of a local (SIRT) and a systemic ( ${ }^{177}$ Lu-PSMA) radionuclide therapy feasibly tolerable in patients with $\mathrm{MCRPC}$ to the liver?

PERTINENT FINDINGS: This retrospectively analyzed patient group indicated that the combination of SIRT and ${ }^{177}$ Lu-PSMA is feasible and effective. Use of ${ }^{177}$ Lu-PSMA alone yields discordant responses by liver metastases, whereas SIRT efficiently targets all liver metastases.

IMPLICATIONS FOR PATIENT CARE: In addition to systemic 177Lu-PSMA therapy, local therapies targeting liver metastases should be evaluated in further clinical studies.

\section{REFERENCES}

1. Halabi S, Kelly WK, Ma H, et al. Meta-analysis evaluating the impact of site of metastasis on overall survival in men with castration-resistant prostate cancer. J Clin Oncol. 2016;34:1652-1659.

2. Kessel K, Seifert R, Schäfers M, et al. Second line chemotherapy and visceral metastases are associated with poor survival in patients with $\mathrm{mCRPC}$ receiving ${ }^{177}$ Lu-PSMA-617. Theranostics. 2019;9:4841-4848.

3. Pouessel D, Gallet B, Bibeau F, et al. Liver metastases in prostate carcinoma: clinical characteristics and outcome. BJU Int. 2007;99:807-811.

4. Wang HT, Yao YH, Li BG, Tang Y, Chang JW, Zhang J. Neuroendocrine prostate cancer (NEPC) progressing from conventional prostatic adenocarcinoma: factors associated with time to development of NEPC and survival from NEPC diagnosis-a systematic review and pooled analysis. J Clin Oncol. 2014;32:3383-3390.

5. Rahbar K, Ahmadzadehfar H, Kratochwil C, et al. German Multicenter study investigating ${ }^{177} \mathrm{Lu}-\mathrm{PSMA}-617$ radioligand therapy in advanced prostate cancer patients. J Nucl Med. 2017;58:85-90.

6. Hofman MS, Violet J, Hicks RJ, et al. [ $\left.{ }^{177} \mathrm{Lu}\right]-P S M A-617$ radionuclide treatment in patients with metastatic castration-resistant prostate cancer (LuPSMA trial): a single-centre, single-arm, phase 2 study. Lancet Oncol. 2018;19:825-833.

7. Bräuer A, Grubert LS, Roll W, et al. ${ }^{177}$ Lu-PSMA-617 radioligand therapy and outcome in patients with metastasized castration-resistant prostate cancer. Eur J Nucl Med Mol Imaging. 2017;44:1663-1670.

8. Rahbar K, Bögeman M, Yordanova A, et al. Delayed response after repeated ${ }^{177}$ Lu-PSMA-617 radioligand therapy in patients with metastatic castration resistant prostate cancer. Eur J Nucl Med Mol Imaging. 2018;45:243-246.

9. Ahmadzadehfar H, Wegen S, Yordanova A, et al. Overall survival and response pattern of castration-resistant metastatic prostate cancer to multiple cycles of radioligand therapy using $\left[{ }^{177} \mathrm{Lu}\right]$ Lu-PSMA-617. Eur J Nucl Med Mol Imaging. 2017;44:1448-1454.

10. Rahbar K, Boegemann M, Yordanova A, et al. PSMA targeted radioligand therapy in metastatic castration resistant prostate cancer after chemotherapy, abiraterone and/or enzalutamide: a retrospective analysis of overall survival. Eur J Nucl Med Mol Imaging. 2018;45:12-19. 
11. Bastiaannet R, Kappadath SC, Kunnen B, Braat AJAT, Lam MGEH, De Jong HWAM. The physics of radioembolization. EJNMMI Phys. 2018.

12. Rahbar K, Bode A, Weckesser M, et al. Radioligand therapy with ${ }^{177}$ Lu-PSMA617 as a novel therapeutic option in patients with metastatic castration resistant prostate cancer. Clin Nucl Med. 2016;41:522-528.

13. Seregni E, Maccauro M, Chiesa $C$, et al. Treatment with tandem $\left[{ }^{90} \mathrm{Y}\right]$ DOTA-TATE and $\left[{ }^{177} \mathrm{Lu}\right]$ DOTA-TATE of neuroendocrine tumours refractory to conventional therapy. Eur J Nucl Med Mol Imaging. 2014;41:223230.

14. Rahbar K, Afshar-Oromieh A, Jadvar H, Ahmadzadehfar H. PSMA theranostics: current status and future directions. Mol Imaging. 2018;17:1536012118776068.

15. Giammarile F, Bodei L, Chiesa C, et al. EANM procedure guideline for the treatment of liver cancer and liver metastases with intra-arterial radioactive compounds. Eur J Nucl Med Mol Imaging. 2011;38:13931406.

16. Fendler WP, Philippe Tiega DB, Ilhan H, et al. Validation of several SUVbased parameters derived from ${ }^{18} \mathrm{~F}$-FDG PET for prediction of survival after SIRT of hepatic metastases from colorectal cancer. J Nucl Med. 2013;54:12021208.
17. Kennedy A, Coldwell D, Sangro B, Wasan H, Salem R. Radioembolization for the treatment of liver tumors: general principles. Am J Clin Oncol Cancer Clin Trials. 2012;35:91-99.

18. Eisenhauer EA, Therasse P, Bogaerts J, et al. New response evaluation criteria in solid tumours: revised RECIST guideline (version 1.1). Eur J Cancer. 2009;45: 228-247.

19. Molenaar JPF, Baten A, Blokx WAM, Hoogendam A. Development of carcinoid tumour in hormonally treated adenocarcinoma of the prostate. Eur Urol. 2009; 56:874-877.

20. Levillain H, Derijckere ID, Marin G, et al. Y-PET/CT-based dosimetry after selective internal radiation therapy predicts outcome in patients with liver metastases from colorectal cancer.EJNMMI Res. 2018:8:60.

21. Delker A, Fendler WP, Kratochwil C, et al. Dosimetry for ${ }^{177}$ Lu-DKFZ-PSMA617: a new radiopharmaceutical for the treatment of metastatic prostate cancer. Eur J Nucl Med Mol Imaging. 2016;43:42-51.

22. Barabasch A, Heinzel A, Bruners P, Kraemer NA, Kuhl CK. Diffusionweighted MRI is superior to PET/CT in predicting survival of patients undergoing ${ }^{90} \mathrm{Y}$ radioembolization of hepatic metastases. Radiology. 2018;288: 764-773. 